\title{
11 Perspektiven der gastroenterologischen Forschung und Lehre
}

\author{
Christian Trautwein, Petra Lynen Jansen, Frank Lammert
}

\section{Aktueller Stand}

Das wichtigste Ziel gastroenterologischer Forschung und Lehre ist, die steigenden Zahlen gastroenterologischer Erkrankungen und die damit einhergehende enorme Belastung für den einzelnen Patienten, aber auch für das Gesundheitssystem insgesamt, zu reduzieren. Die aktuelle gastroenterologische Forschungslandschaft bietet dafür exzellente strukturelle Voraussetzungen. Die Gastroenterologie ist an allen 36 deutschen staatlichen Universitäten mit Medizinischen Fakultäten vertreten. In der Regel ist dies mit einer eigenständigen, bettenführenden Abteilung verbunden. Gemessen am seit 1996 erfassten h-index rangiert die deutsche Gastroenterologie international hinter den USA, Japan und China auf Platz 4 [1].

Die steigende Zahl von Einzelförderungsanträgen bei der Deutschen Forschungsgemeinschaft sowohl für Forschungsvorhaben in der Gastroenterologie als auch in der Hämatoonkologie, die die Anträge zur Erforschung der malignen Erkrankungen des Magen-Darm-Traktes, der Leber und des Pankreas miterfasst, untermauert die hohe Forschungsaktivität des Fachgebiets [2] (Abb. 11.1). Im Zeitraum zwischen 2005 und 2018 hat sich die Zahl der pro Jahr positiv beschiedenen Anträge aus der Gastroenterologie nahezu verdoppelt (69 versus 131 Anträge, Abb.11.1). Kritisch muss gesehen werden, dass deutlich mehr DFG-Anträge aus der Hämatoonkologie bewilligt werden als aus der Gastroenterologie. Dies könnte ein Indiz dafür sein, dass die Krebsforschung im Gegensatz zur Erforschung nicht-maligner Erkrankungen bevorzugt gefördert wird.

Ebenfalls herausragend ist die durch die DFG begutachtete Verbundförderung. Aktuell kann die universitäre Gastroenterologie insgesamt sieben Sonderforschungsbereiche und zwei Exzellenzcluster vorweisen [3] (Tab. 11.1).

Durch die deutschlandweite Verteilung dieser Verbundprojekte sind prinzipiell über 40 Millionen Menschen für die Einbeziehung in klinische Studien beziehungsweise für die Translation neuester wissenschaftlicher Erkenntnisse in die klinische Praxis erreichbar (Abb.11.2).

Bei der BMBF-Förderung und der Förderung durch den G-BA ist die Gastroenterologie gleichermaßen erfolgreich. In den beiden Kompetenzzentren Leber (Hep-Net) und chronisch entzündliche Darmerkrankungen (CED) wurden Strukturen für die Umsetzung klinischer Studien etabliert. Es existieren derzeit sieben geförderte Innovationsfondsprojekte (Tab. 11.2), vier weitere sind aktuell beantragt [4]. Gemeinsames Merkmal der gastroenterologischen Innovationsfondsprojekte ist, dass sie stark auf die Prävention ausgerichtet sind. So sollen mit dem Projekt FARKOR Menschen mit einem familiär erhöhten Darmkrebsrisiko bereits früh identifiziert werden. Ziel ist es, dieser besonders jungen Risikogruppe, in der die Darmkrebsinzidenz derzeit jähr- 
Tab. 11.1: Verbundprojekte und Exzellenzcluster der Gastroenterologie.

\begin{tabular}{|c|c|c|c|}
\hline Abkürzung & Titel & Sprecher & Hochschule \\
\hline SFB 738 & $\begin{array}{l}\text { Optimierung kon- } \\
\text { ventioneller und } \\
\text { innovativer Trans- } \\
\text { plantate }\end{array}$ & $\begin{array}{l}\text { Prof. Dr. Michael Peter Manns } \\
\text { Klinik für Gastroenterologie, } \\
\text { Hepatologie und Endokrinologie } \\
\text { Medizinische Hochschule } \\
\text { Hannover }\end{array}$ & $\begin{array}{l}\text { Medizinische Hochschule } \\
\text { Hannover }\end{array}$ \\
\hline SFB 841 & $\begin{array}{l}\text { Leberentzündungen: } \\
\text { Infektion, Immun- } \\
\text { regulation und Kon- } \\
\text { sequenzen }\end{array}$ & $\begin{array}{l}\text { Prof. Dr. Ansgar W. Lohse } \\
\text { Eppendorf } \\
\text { I. Medizinische Klinik und Polikli- } \\
\text { nik Universitätsklinikum Hamburg }\end{array}$ & $\begin{array}{l}\text { Universität Hamburg } \\
\text { Christian-Albrechts- } \\
\text { Universität zu Kiel }\end{array}$ \\
\hline SFB 974 & $\begin{array}{l}\text { Kommunikation und } \\
\text { Systemrelevanz bei } \\
\text { Leberschädigung } \\
\text { und Regeneration }\end{array}$ & $\begin{array}{l}\text { Prof. Dr. Dieter Häussinger } \\
\text { Klinik für Gastroenterologie, } \\
\text { Hepatologie, und Infektiologie } \\
\text { Universitätsklinikum Düsseldorf }\end{array}$ & $\begin{array}{l}\text { Heinrich-Heine-Universität } \\
\text { Düsseldorf } \\
\text { Universitätsklinikum Essen }\end{array}$ \\
\hline SFB 1321 & $\begin{array}{l}\text { Modellierung und } \\
\text { Targeting des Pan- } \\
\text { kreaskarzinoms }\end{array}$ & $\begin{array}{l}\text { Prof. Dr. Roland M. Schmid } \\
\text { Klinik und Poliklinik für Innere } \\
\text { Medizin II } \\
\text { Technische Universität München }\end{array}$ & $\begin{array}{l}\text { Technische Universität } \\
\text { München } \\
\text { Ludwig-Maximilians- } \\
\text { Universität München }\end{array}$ \\
\hline SFB 1371 & $\begin{array}{l}\text { Microbiome Sig- } \\
\text { natures - Funk- } \\
\text { tionelle Relevanz } \\
\text { des Mikrobioms im } \\
\text { Verdauungstrakt }\end{array}$ & $\begin{array}{l}\text { Professor Dr. Dirk Haller } \\
\text { Technische Universität München } \\
\text { Zentralinstitut für Ernährungs- } \\
\text { und Lebensmittelforschung } \\
\text { Lehrstuhl für Ernährung und } \\
\text { Immunologie }\end{array}$ & $\begin{array}{l}\text { Ludwig-Maximilians-Uni- } \\
\text { versität München } \\
\text { Technische Universität } \\
\text { Dresden } \\
\text { Universitätsklinikum } \\
\text { Regensburg } \\
\text { Universitätsklinikum } \\
\text { Aachen } \\
\text { Medizinische Hochschule } \\
\text { Hannover }\end{array}$ \\
\hline SFB/TRR 57 & $\begin{array}{l}\text { Organfibrose: Von } \\
\text { den Mechanismen } \\
\text { der Schädigung zur } \\
\text { Beeinflussung der } \\
\text { Erkrankung }\end{array}$ & $\begin{array}{l}\text { Prof. Dr. Christian Trautwein } \\
\text { Klinik für Gastroenterologie } \\
\text { Uniklinik der Rheinisch-West- } \\
\text { fälisch Technischen Hochschule } \\
\text { Aachen }\end{array}$ & $\begin{array}{l}\text { Rheinisch-Westfälische } \\
\text { Technische Hochschule } \\
\text { Aachen } \\
\text { Rheinische Friedrich- } \\
\text { Wilhelms-Universität Bonn } \\
\text { Ludwig-Maximilians- } \\
\text { Universität München }\end{array}$ \\
\hline $\begin{array}{l}\text { SFB/TRR } \\
241\end{array}$ & $\begin{array}{l}\text { Immunepitheliale } \\
\text { Signalwege bei chro- } \\
\text { nisch-entzündlichen } \\
\text { Darmerkrankungen }\end{array}$ & $\begin{array}{l}\text { Prof. Dr. Christoph Becker } \\
\text { Medizinische Klinik I } \\
\text { Universitätsklinikum der } \\
\text { Friedrich-Alexander-Universität } \\
\text { Erlangen-Nürnberg }\end{array}$ & $\begin{array}{l}\text { Friedrich-Alexander- } \\
\text { Universität Erlangen- } \\
\text { Nürnberg } \\
\text { Charité Berlin }\end{array}$ \\
\hline
\end{tabular}


Tab. 11.1: (fortgesetzt) Verbundprojekte und Exzellenzcluster der Gastroenterologie.

\begin{tabular}{|c|c|c|c|}
\hline Abkürzung & Titel & Sprecher & Hochschule \\
\hline EXC & $\begin{array}{l}\text { Präzisionsmedizin } \\
\text { für chronische Ent- } \\
\text { zündungserkran- } \\
\text { kungen }\end{array}$ & $\begin{array}{l}\text { Prof. Dr. Stefan Schreiber } \\
\text { Klinik für Innere Medizin I } \\
\text { Christian-Albrechts-Universität } \\
\text { zu Kiel }\end{array}$ & $\begin{array}{l}\text { Christian-Albrechts- } \\
\text { Universität zu Kiel }\end{array}$ \\
\hline EXC & $\begin{array}{l}\text { Individualisierung } \\
\text { von Tumortherapien } \\
\text { durch molekulare } \\
\text { Bildgebung und } \\
\text { funktionelle Identi- } \\
\text { fizierung therapeuti- } \\
\text { scher Zielstrukturen } \\
\text { (iFIT) }\end{array}$ & $\begin{array}{l}\text { Prof. Dr. Lars Zender } \\
\text { Universitätsklinik für Innere } \\
\text { Medizin VIII } \\
\text { Universität Tübingen }\end{array}$ & $\begin{array}{l}\text { Eberhard-Karls-Universität } \\
\text { Tübingen }\end{array}$ \\
\hline
\end{tabular}

\section{DFG-Einzelförderung Gastroenterologie}

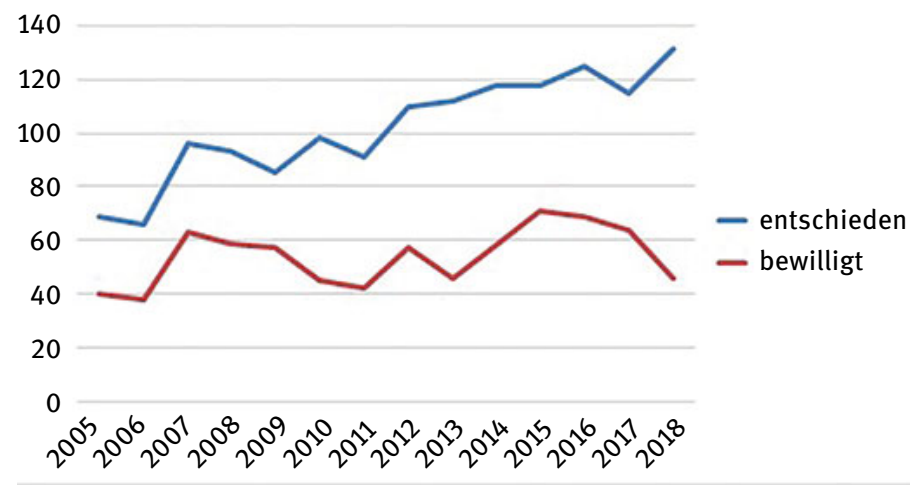

DFG-Einzelförderung Hämatoonkologie

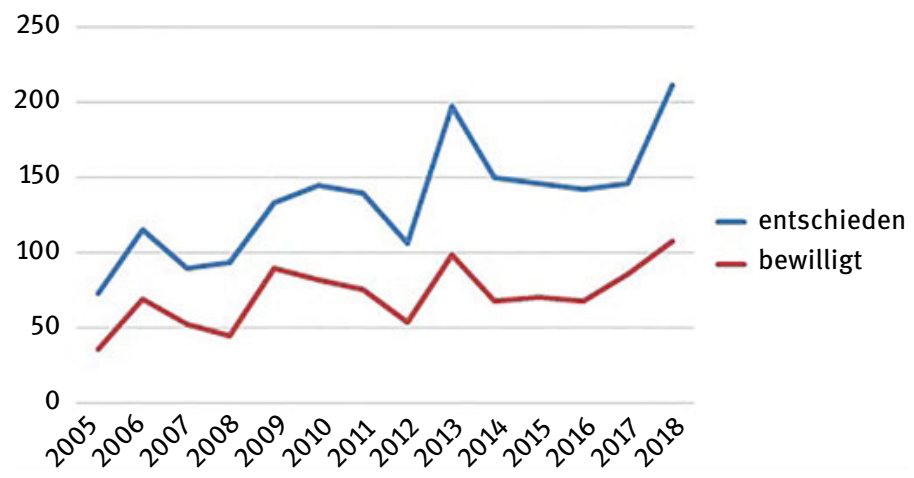

Abb. 11.1: DFGEinzelförderung 2005-2018 (Quelle: DFG). 


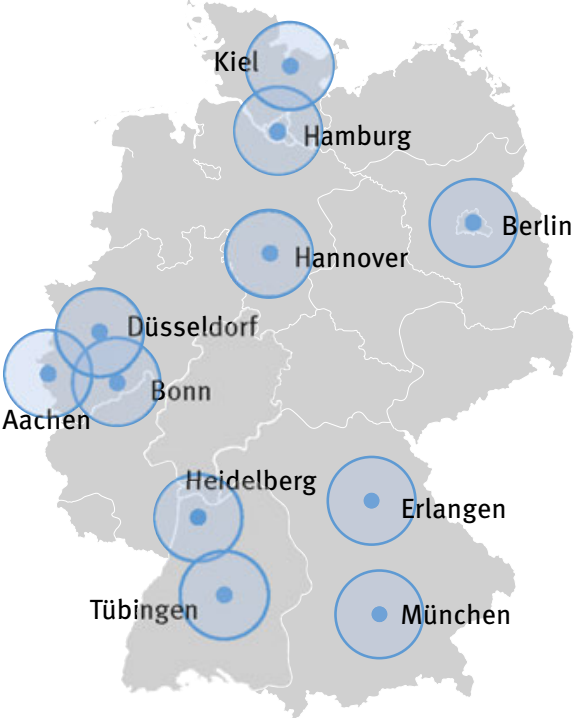

\begin{tabular}{|c|c|c|}
\hline \multicolumn{2}{|c|}{$\begin{array}{l}\text { Einwohner } \\
\text { Umkreis } 50 \text { km }\end{array}$} & \\
\hline Kiel & 979.000 & \\
\hline Berlin & 4.461 .600 & \\
\hline Düsseldorf & 8.646 .900 & Abb. 11.2: Regionale \\
\hline Bonn & 6.227 .400 & $\begin{array}{l}\text { Verteilung der gas- } \\
\text { troenterologischen }\end{array}$ \\
\hline Heidelberg & 3.539 .000 & Sonderforschungsbe- \\
\hline München & 3.062 .200 & $\begin{array}{l}\text { reiche und potenzielle } \\
\text { Einzugsgebiete. }\end{array}$ \\
\hline
\end{tabular}

lich um 2\% steigt, eine risikoangepasste Darmkrebsvorsorge anzubieten. Das Projekt SEAL (Strukturierte Früh-Erkennung einer Asymptomatischen Leberzirrhose) erprobt, inwieweit ein Leberwert-Screening beim Hausarzt dabei hilft, mehr Patienten mit chronischen Leberkrankheiten frühzeitig zu diagnostizieren und wirksam zu behandeln.

Die DGVS hat 26 hochwertige, evidenzbasierte Leitlinien erarbeitet, die kontinuierlich aktualisiert werden und mit denen eine Implementierung wissenschaftlicher Erkenntnisse in die klinische Praxis unmittelbar gewährleistet ist (Leitlinien der DGVS, Stand: März 2019 (S.281/282)). Der Gastroenterologie ist es ein wichtiges Anliegen, ihre Wissensbasis möglichst umfassend auch jungen Ärzten und Wissenschaftlern zur Verfügung zu stellen. Aus diesem Grunde sind alle Leitlinien der DGVS nicht nur über die DGVS Homepage frei verfügbar, sondern werden auch in andere Wissensdatenbanken integriert. Dieses Ziel wird derzeit in einer Kooperation mit dem insbesondere von Studierenden viel genutzten Portal Amboss umgesetzt.

Die DGVS-Nachwuchskampagne zielt darauf ab, junge Ärztinnen und Ärzte für die Gastroenterologie zu gewinnen. Unter www.gastroenterologe-werden.de können sich Studierende über Inhalte und Möglichkeiten der gastroenterologischen Weiterbildung informieren, PJ-Stellen finden oder Präsentationen $\mathrm{zu}$ wichtigen gastroenterologischen Themen herunterladen. Mit der Tauschbörse Lehre ist ein Portal entstanden, das den Austausch von hervorragendem Lehrmaterial unter den Lehrenden in der Gastroenterologie ermöglicht. Hinzu kommen die jährlich vergebenen, zahlreichen Preise und Promotionsstipendien, mit denen die DGVS besonders den wissenschaftlichen Nachwuchs fördert. 
Tab. 11.2: Innovationsfondsprojekte in der Gastroenterologie.

\begin{tabular}{|c|c|c|}
\hline Abkürzung & Titel & Partner \\
\hline $\begin{array}{l}\text { CED BIO-AS- } \\
\text { SIST }\end{array}$ & $\begin{array}{l}\text { Bessere Versorgung von Patienten mit chronisch-ent- } \\
\text { zündlichen Darmerkrankungen durch die Delegation } \\
\text { von ärztlichen Leistungen an eine Biologika-Nurse }\end{array}$ & $\begin{array}{l}\text { PD Dr. B. Bokemeyer } \\
\text { Kompetenznetz Darm- } \\
\text { erkrankungen, Minden }\end{array}$ \\
\hline $\begin{array}{l}\text { CED-KQN - Big } \\
\text { Data-eHealth }\end{array}$ & $\begin{array}{l}\text { Verbesserung der Versorgung von Kindern und Jugend- } \\
\text { lichen mit chronisch-entzündlichen Darmerkrankungen }\end{array}$ & $\begin{array}{l}\text { PD Dr. Jan de Laffolie } \\
\text { Justus-Liebig-Universität, } \\
\text { Gießen }\end{array}$ \\
\hline EDIUM & $\begin{array}{l}\text { Ergebnisqualität bei Darmkrebs: Identifikation von } \\
\text { Unterschieden und Maßnahmen zur flächendeckenden } \\
\text { Qualitätsentwicklung }\end{array}$ & $\begin{array}{l}\text { Dr. Christoph Kowalski } \\
\text { Deutsche Krebsgesell- } \\
\text { schaft e. V., Berlin }\end{array}$ \\
\hline FARKOR & $\begin{array}{l}\text { Vorsorge bei familiärem Risiko für das kolorektale } \\
\text { Karzinom }\end{array}$ & $\begin{array}{l}\text { Patrizia Ungar } \\
\text { Kassenärztliche Ver- } \\
\text { einigung Bayerns (KVB), } \\
\text { München }\end{array}$ \\
\hline KOL-OPT_UH & $\begin{array}{l}\text { Fehlversorgung bzgl. Kontroll-Koloskopien in Deutsch- } \\
\text { land: Ausmaß, Determinanten und Konzipierung von } \\
\text { Lösungsansätzen }\end{array}$ & $\begin{array}{l}\text { Prof. Dr. Ulrike Haug } \\
\text { Leibniz-Institut für Präven- } \\
\text { tionsforschung und Epi- } \\
\text { demiologie, Bremen }\end{array}$ \\
\hline SEAL & $\begin{array}{l}\text { Strukturierte Früh-Erkennung einer asymptomatischen } \\
\text { Leberzirrhose in Rheinland-Pfalz und im Saarland }\end{array}$ & $\begin{array}{l}\text { Prof. Dr. Peter Galle und } \\
\text { Prof. Dr. Frank Lammert } \\
\text { I. Medizinische Klinik und } \\
\text { Poliklinik, Universitäts- } \\
\text { medizin Mainz und Klinik } \\
\text { für Innere Medizin II, } \\
\text { Universitätsklinikum des } \\
\text { Saarlandes }\end{array}$ \\
\hline SIGMO & $\begin{array}{l}\text { Die Sigmoidoskopie als evidenzbasiertes Screening- } \\
\text { verfahren für Darmkrebs - eine mögliche Option? }\end{array}$ & $\begin{array}{l}\text { Dr. Maren Dreier } \\
\text { Institut für Epidemiolo- } \\
\text { gie, Sozialmedizin und } \\
\text { Gesundheitssystem- } \\
\text { forschung, Medizinische } \\
\text { Hochschule Hannover }\end{array}$ \\
\hline
\end{tabular}

Diese Aktivitäten haben dazu beigetragen, die Mitgliederzahlen der DGVS, und dabei insbesondere die Anzahl junger Mitglieder unter 35 Jahren, deutlich zu steigern. Die DGVS hat aktuell über 6.000 aktive Mitglieder (Abb. 11.3).

Im Jahre 2019 wurde außerdem die Kampagne Gesundheit durch Gastroenterologie (www.gesundheit-durch-gastroenterologie.de) gestartet. Ziel des Projekts ist es, der Öffentlichkeit die Bedeutung gastroenterologischer Erkrankungen und ihren Status als relevante Volkskrankheiten näherzubringen. Dadurch soll Bedeutung und Relevanz gastroenterologischer Wissenschaft deutlich gemacht und geschärft werden 


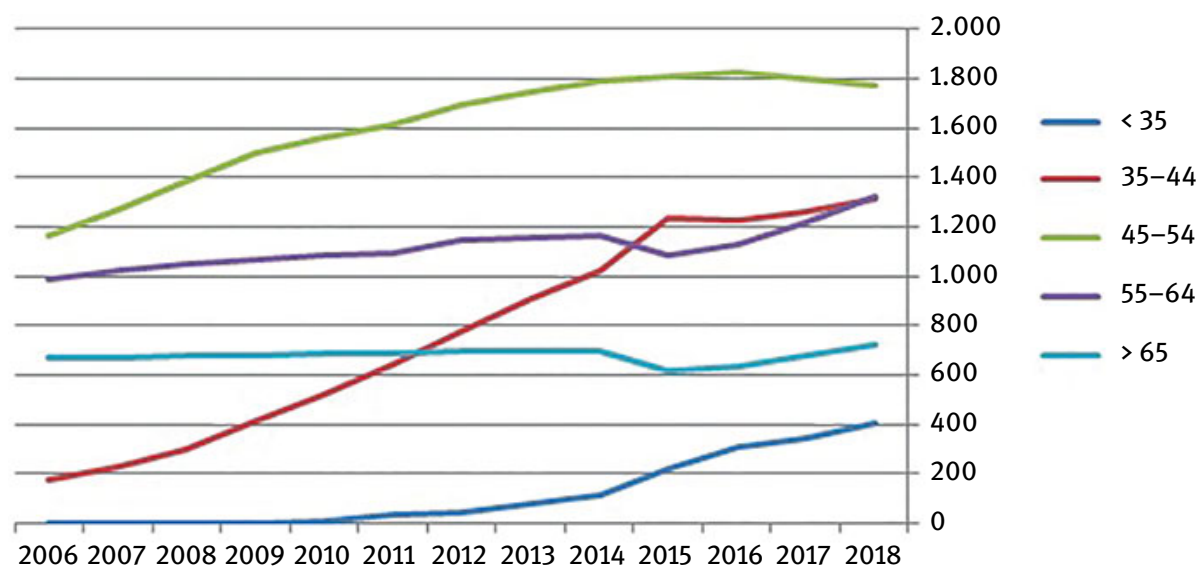

Abb. 11.3: Entwicklung der Mitgliederzahlen der DGVS.

und so auch Eingang in Überlegungen der zukünftigen Gesundheits- und Forschungspolitik finden.

\section{Zukunftsperspektiven}

Ein besonderes Merkmal gastroenterologischer Erkrankungen ist die Kaskade chronische Entzündung, Fibrose (Vernarbung) mit entsprechendem Funktionsausfall des betroffenen Organs bis hin zur malignen Entartung (Krebsentstehung). Beispiele hierfür sind die Fettleberhepatitis, die chronisch entzündlichen Darmerkrankungen oder die Entzündung der Bauchspeicheldrüse (Pankreatitis). All diese Erkrankungen können unbehandelt zu Krebserkrankungen in den entsprechenden Organen führen (Leberkrebs, Darmkrebs, Bauchspeicheldrüsenkrebs). Der Verlauf dieser Kaskade wird durch genetische Faktoren und/oder Umweltfaktoren sowie den Lifestyle (Ernährung, Alkohol, Bewegung) unmittelbar beeinflusst.

Über die Leber-Darm-Achse steuert der Magen-Darm-Trakt direkt den Energiehaushalt des Körpers. Daher haben Veränderungen in der Homöostase des Gastrointestinaltrakts (z. B. Fehlernährung, chronische Entzündungsvorgänge, Veränderungen der Darmflora) einen unmittelbaren Einfluss auf andere Krankheitsbilder. Dies gilt unter anderem für Diabetes, Herz-Kreislauf-Erkrankungen oder neurologische Erkrankungen. Zusammenfassend kommt damit dem Magen-Darm-Trakt die entscheidende „Gate-keeper-Funktion“ für die menschliche Gesundheit in einer sich rasant verändernden Industriegesellschaft zu.

Daher trägt gastroenterologische Forschung unmittelbar zum Verständnis organspezifischer und organübergreifender Mechanismen, sowie zum Verständnis der Kommunikation zwischen den verschiedenen Organen des Körpers, bei. Dies hat unmittelbar Einfluss auf die Implementierung neuer, gezielter, aber auch übergrei- 
fender Therapieprinzipien. Darüber hinaus können durch neue molekulare Erkenntnisse Rückschlüsse auf neue Präventionsstrategien gezogen werden. Aufgrund der Relevanz gastroenterologischer Wissenschaft für die Erforschung neuer organübergreifender pathophysiologischer Konzepte, stellt die Gastroenterologie ein zentrales Fach dar, um integraler Bestandteil übergreifender wissenschaftlicher Netzwerksstrukturen zu sein.

Ein besonderes Anliegen der DGVS ist der Aufbau einer Präventionsstruktur für Deutschland. Die Medizin der letzten Jahrzehnte ist vor allem durch eine Kultur des Reparierens auf der Endstrecke des Lebens gekennzeichnet. Die meisten Kosten im Gesundheitssystem fallen in den letzten zwölf Monaten des Lebens an. Innovative Ansätze in einer alternden Gesellschaft müssen früher ansetzen und darauf abzielen, rechtzeitig mögliche Krankheitsrisiken zu erkennen und diese gezielt über Maßnahmen der Primär- und Sekundärprävention zu verhindern. Die Gastroenterologie hat über die Einführung der Koloskopie oder der Hepatitis-B-Impfung schon wichtige Präventionsmaßnahmen klinisch implementiert.

Um diese erfolgreichen Ansätze weiter auszubauen, fordert die DGVS die Etablierung eines übergreifenden Nationalen Präventionszentrums (NPZ) unter Einbindung möglichst vieler Partner. Das NPZ soll modular aufgebaut sein, um - beginnend mit einer Kernstruktur - kontinuierlich eine wissenschaftliche Netzstruktur zu entwickeln, die dann zu neuen Präventionsansätzen im Sinne des Patienten und der gesamten Gesellschaft (medizinisch und ökonomisch) führt. Initial könnte dabei eine Vernetzungsstruktur gemeinsam mit den bestehenden deutschen Gesundheitszentren gebildet werden, wobei im Verlauf weitere Partner, wie Krankenkassen, Patienten- und Sozialverbände, Schulen, Apotheken, Psychologen und andere, eingebunden werden sollten, um darüber eine direkte Interaktion von der Forschung bis zum Patienten zu erreichen. Daher fordert die DGVS hier die nachhaltige Unterstützung der Politik, um das Konzept des Nationalen Präventionszentrums zum Wohle der Patienten implementieren zu können.

\section{Literatur}

[1] Scimago journal und country rank. www.scimagojr.com/countryrank. Zugriff: 29.03.2019.

[2] Deutsche Forschungsgemeinschaft (DFG). Informationsmanagement Statistik und Reporting. Förderquotenstatistik. Stand 22.09.2016.

[3] Deutsche Forschungsgemeinschaft (DFG). Gepris Datenbank. http://gepris.dfg.de/gepris. Zugriff: 29.03.2019.

[4] Gemeinsamer Bundesausschuss. Innovationsfondprojekte. https://innovationsfonds.g-ba.de/ projekte/. Zugriff: 29.03.2019. 
